TecnoLógicas

ISSN-p 0123-7799

ISSN-e 2256-5337

Vol. 21, No. 41, pp. 115-134

Enero-abril de 2018

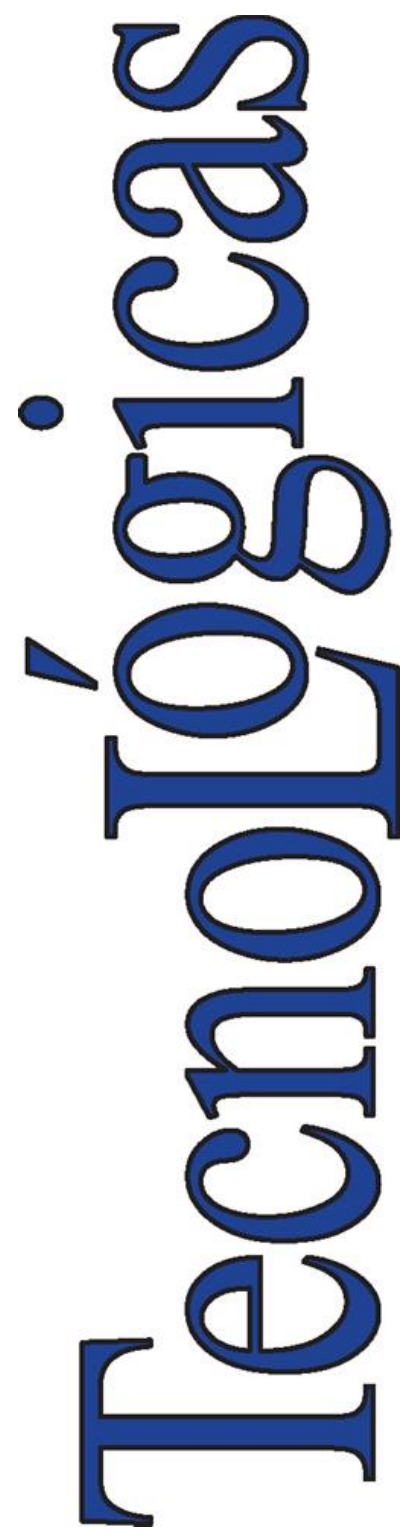

(C) Copyright 2015 por autores y Tecno Lógicas Este trabajo está licenciado bajo una Licencia Internacional Creative Commons Atribución (CC BY)

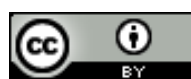

Artículo de Revisión/Review Article

\section{El trabajo colaborativo como estrategia didáctica para la enseñanza/aprendizaje de la programación: una revisión sistemática de literatura}

\section{Collaborative work as a didactic strategy for teaching/learning programming: a systematic literature review}

\author{
Oscar Revelo-Sánchez ${ }^{1}$, César A. Collazos-Ordóñez ${ }^{2}$ \\ y Javier A. Jiménez-Toledo ${ }^{3}$
}

Recibido: 26 de septiembre de 2017

Aceptado: 11 de noviembre de 2017

Cómo citar / How to cite

O. Revelo-Sánchez, C. A. Collazos-Ordoñez, y J. A. Jiménez-Toledo, El trabajo colaborativo como estrategia didáctica para la enseñanza/aprendizaje de la programación: una revisión sistemática de literatura. TecnoLógicas, vol. 21, no. 41, pp. 115-134, 2018.

1 MSc en Investigación de Operaciones, Departamento de Sistemas, Universidad de Nariño, Pasto-Colombia, orevelo@udenar.edu.co

$2 \mathrm{PhD}$ en Ciencias, Departamento de Sistemas, Universidad del Cauca, Popayán-Colombia, ccollazo@unicauca.edu.co

3 MSc en Computación, Facultad de Ingeniería, Institución Universitaria CESMAG, Pasto-Colombia, jajimenez@iucesmag.edu.co 
El trabajo colaborativo como estrategia didáctica para la enseñanza/aprendizaje de la programación: una revisión sistemática de literatura

\title{
Resumen
}

La incorporación del trabajo colaborativo en cursos de programación ha sido identificada como una estrategia potencial que podría maximizar la participación de los estudiantes y tener un impacto positivo en el aprendizaje. En las fuentes consultadas no se ha encontrado un estudio dirigido a recopilar y analizar los resultados de investigación sobre este tema utilizando un método sistemático. Para tratar de llenar este vacío, se realizó una revisión sistemática de literatura con el objetivo de resumir los estudios sobre el uso del trabajo colaborativo como estrategia didáctica para la enseñanza/aprendizaje de la programación. Inicialmente, mediante la búsqueda en cuatro bases de datos de publicaciones científicas, se obtuvieron 95 estudios publicados en los últimos cinco años. Luego de un cuidadoso análisis de cada uno de ellos, se verifica que 40 coinciden con los requerimientos de la revisión, análisis que dio como resultado la síntesis de once Técnicas de Aprendizaje Colaborativo (TAC) que llevan a la práctica la estrategia, que a su vez agrupan a 19 denominaciones comunes de estrategias encontradas en los documentos, es decir, las estrategias o técnicas colaborativas asociadas a cada estudio. La revisión arrojó también una cantidad importante de aportes de la comunidad investigadora que sientan una base importante para trabajos futuros, dejando en claro que el trabajo colaborativo se consolida cada vez más como una estrategia didáctica válida y pertinente, no solo en la enseñanza/aprendizaje de la programación, sino también en otras áreas del conocimiento incluyendo las ciencias de la computación.

\section{Palabras clave}

Aprendizaje colaborativo, enseñanza/aprendizaje, programación, revisión sistemática, trabajo colaborativo.

\begin{abstract}
The incorporation of Collaborative Work into programming courses has been identified as a potential strategy that could maximize student participation and have a positive impact on learning. In the consulted sources, no study has been found to collect and analyze the results of research on this subject using a systematic method. To try to fill this gap, a systematic literature review was conducted with the aim of summarizing the studies on the use of Collaborative Work as a didactic strategy for teaching/learning programming. Initially, through a search in four (4) databases of scientific publications, 95 studies published in the last five (5) years were obtained. After careful analysis of each one of them, only 40 were found to meet the review requirements. This analysis resulted in the synthesis of eleven (11) Collaborative Learning Techniques (CLT) that implement such strategy. Subsequently, they were grouped into 19 common names of strategies found in the documents, i.e. the collaborative strategies or techniques associated with each study. The review also showed a significant amount of contributions from the research community that constitute an important basis for future work. This demonstrates that Collaborative Work is increasingly consolidated as a valid and relevant didactic strategy, not only in programming teaching/learning, but also in other areas of knowledge including computer science.
\end{abstract}

\section{Keywords}

Collaborative Learning, Teaching/Learning, Programming, Systematic Review, Collaborative Work. 
El trabajo colaborativo como estrategia didáctica para la enseñanza/aprendizaje de la programación: una revisión sistemática de literatura

\section{INTRODUCCIÓN}

El trabajo colaborativo es un proceso en el que un individuo aprende más de lo que aprendería por sí solo, fruto de la interacción de los integrantes de un equipo, quienes saben diferenciar y contrastar sus puntos de vista, de tal manera, que llegan a generar un proceso de construcción de conocimiento [1]. La consecuencia de este proceso es lo que se conoce como aprendizaje colaborativo [2]. La incorporación en el aula del trabajo colaborativo como una estrategia didáctica, que redunde en un aprendizaje colaborativo, requiere de la utilización de técnicas que lleven a la práctica la estrategia. En [3] las han denominado Técnicas de Aprendizaje Colaborativo (TAC), formas comunes de estructurar las interacciones entre los participantes en diferentes actividades de aprendizaje colaborativo, así como la información que se intercambia y los objetos que se manipulan [4].

El objetivo de este artículo es presentar el resultado de una revisión sistemática de literatura, en la que se elaboró una síntesis del trabajo colaborativo reportado en publicaciones científicas como una estrategia didáctica para la enseñanza/aprendizaje de la programación. Particularmente, se analizaron los aspectos generales de los documentos revisados $\mathrm{y}$, a nivel de contenido, las TAC involucradas en los diferentes estudios. El análisis de los aspectos generales se enfocó en la identificación de la ubicación geográfica y los tipos de publicaciones. Por su parte, en el análisis de contenido se buscaron respuestas a las preguntas de interés que se enumeran a continuación.

RQ1: ¿Cuáles son los enfoques de aprendizaje y los tipos de contribuciones de los estudios sobre el trabajo colaborativo como estrategia didáctica para la enseñanza/aprendizaje de la programación?

RQ2: ¿Cuáles son las técnicas colaborativas más comunes que han sido definidas en la literatura científica?
RQ3: ¿Cuáles son los propósitos de las técnicas colaborativas más comunes, empleadas en la enseñanza/aprendizaje de la programación?

RQ4: ¿Qué piensan los autores acerca del trabajo colaborativo como estrategia didáctica para la enseñanza/aprendizaje de la programación?

Para presentar esta revisión sistemática de literatura, se definió la siguiente estructura: en la Sección 1 se realiza una breve introducción a la temática y al documento; en la Sección 2 se brinda una fundamentación teórica sobre el trabajo colaborativo, con una visión taxonómica, para llegar a una clasificación de las técnicas colaborativas identificadas en los diferentes estudios; en la Sección 3, se describe el método de investigación utilizado; en la Sección 4, se presentan los resultados obtenidos; en la Sección 5, se presenta la discusión; y en la Sección 6, se presentan las conclusiones; por último, se enumeran las referencias correspondientes.

\section{FUNDAMENTACIÓN TEÓRICA}

El trabajo colaborativo, en un contexto educativo, constituye un modelo de aprendizaje interactivo, que invita a los estudiantes a construir juntos, lo cual demanda conjugar esfuerzos, talentos y competencias, mediante una serie de transacciones que les permitan lograr las metas establecidas consensuadamente. Más que una técnica, el trabajo colaborativo es considerado una filosofía de interacción y una forma personal de trabajo, que implica el manejo de aspectos, tales como el respeto a las contribuciones individuales de los miembros del grupo [5].

En el Tabla 1 se presentan algunas de las conceptualizaciones sobre trabajo colaborativo que se encuentran en la literatura especializada, con el propósito de apreciar sus aspectos constitutivos; entre los que más se destacan están la autoridad, la negociación y los procesos de diálogo que se 
El trabajo colaborativo como estrategia didáctica para la enseñanza/aprendizaje de la programación: una revisión sistemática de literatura

dan al interior del grupo, la reciprocidad, la responsabilidad y las relaciones sociales.

Para [10], el trabajo colaborativo posee una serie de características que lo diferencian del trabajo en grupo y de otras modalidades de organización grupal. Algunas de ellas son las siguientes:

Se encuentra basado en una fuerte relación de interdependencia entre los diferentes miembros del grupo, de manera que el alcance final de las metas concierna a todos sus miembros.

Hay una clara responsabilidad individual de cada miembro del grupo para el alcance de la meta final.

La formación de los grupos en el trabajo colaborativo es heterogénea en habilidades y características de los miembros; en el trabajo tradicional de grupos, estas son más homogéneas.

Todos los miembros tienen su parte de responsabilidad para la ejecución de las acciones en el grupo.

La responsabilidad de cada miembro del grupo es compartida.
Se persigue el logro de objetivos a través de la realización (individual y conjunta) de tareas.

Existe una interdependencia positiva entre los sujetos.

El trabajo colaborativo exige de los participantes habilidades comunicativas, relaciones simétricas y recíprocas, así como un deseo de compartir la resolución de las tareas.

Según [2], para poder entender el concepto de trabajo colaborativo como metodología, es preciso detenerse en el encuadre teórico que sustenta este modelo de enseñanza. Si se parte de la idea de que un proceso pedagógico está impregnado de habilidades sociales y que la comunicación es inherente en todo grupo humano, la construcción colectiva de los aprendizajes a través del diálogo se mantiene presente a través de los tiempos. Trabajar en el aula, para fines comunes, utilizando las estrategias adecuadas, hace que los estudiantes vayan desarrollando estrategias interpersonales y altos niveles de pensamiento para un profundo conocimiento del contenido.

Tabla 1. Conceptualizaciones de trabajo colaborativo. Fuente: autores.

\begin{tabular}{ll}
\hline \multicolumn{1}{c}{ Autores } & \multicolumn{1}{c}{ Conceptualizaciones } \\
\hline $\begin{array}{l}\text { Guitert y Giménez, } \\
1997 \text { [1] }\end{array}$ & $\begin{array}{l}\text { Proceso en el que cada individuo aprende más de lo que aprendería por sí solo, fruto de la interacción } \\
\text { de los integrantes del equipo. El trabajo colaborativo se da cuando existe una reciprocidad entre un } \\
\text { conjunto de individuos que saben diferenciar y contrastar sus puntos de vista de tal manera que } \\
\text { llegan a generar un proceso de construcción de conocimiento. }\end{array}$ \\
\hline $\begin{array}{l}\text { Panitz y Panitz, 1998 } \\
\text { [6] }\end{array}$ & $\begin{array}{l}\text { Proceso de interacción cuya premisa básica es la construcción de consenso. Se comparte la autoridad y } \\
\text { entre todos se acepta la responsabilidad de las acciones del grupo. }\end{array}$ \\
\hline Gros, 2000 [7] & $\begin{array}{l}\text { Proceso en el que las partes se comprometen a aprender algo juntas. Lo que debe ser aprendido solo } \\
\text { puede conseguirse si el trabajo del grupo es realizado en colaboración. Es el grupo el que decide cómo } \\
\text { realizar la tarea, qué procedimientos adoptar, y cómo dividir el trabajo o tareas a realizar. La comuni- } \\
\text { cación y la negociación son claves en este proceso. }\end{array}$ \\
\hline Salinas, 2000 [8] & $\begin{array}{l}\text { Considera fundamental el análisis de la interacción profesor-estudiante y estudiante-estudiante, por } \\
\text { cuanto el trabajo busca el logro de metas de tipo académico y también la mejora de las propias rela- } \\
\text { ciones sociales. }\end{array}$ \\
\hline Lucero, 2003 [9] & $\begin{array}{l}\text { Conjunto de métodos de instrucción y entrenamiento apoyados con estrategias para propiciar el } \\
\text { desarrollo de habilidades mixtas (aprendizaje y desarrollo personal y social), donde cada miembro del } \\
\text { grupo es responsable tanto de su aprendizaje como del de los demás miembros del grupo. }\end{array}$ \\
\hline
\end{tabular}


El trabajo colaborativo como estrategia didáctica para la enseñanza/aprendizaje de la programación: una revisión sistemática de literatura

De lo anterior, surge el constructo de aprendizaje colaborativo. Este se sustenta en enfoques cognitivistas. Si se parte de una enseñanza centrada en el estudiante, cómo promueve el constructivismo, se tiene cuenta lo que pasa al interior de cada aprendiz, pero también la forma conjunta y social de promover los aprendizajes nuevos y engancharlos con los que ya existen para crear una estructura de pensamiento cada vez más pertinente con los procesos de enseñanza y aprendizaje. No solo importa el producto; son importantes también los procedimientos y rutas para la consecución de nuevos saberes. De ahí la referencia al constructivismo social como el eje central de este enfoque. Sin embargo, cabe destacar que no se trata solamente de un enfoque centrado en el estudiante. De acuerdo con [10], se requiere reforzar y entender la necesidad de "desplazar la responsabilidad de los aprendizajes a los propios estudiantes que necesariamente pasa a convertirlos en sujetos activos de la construcción y gestión de su propio conocimiento". Es, ir estimulando la autogestión de los conocimientos y el control de los aprendizajes en manos de los mismos estudiantes.

El aprendizaje colaborativo según [11] es un sistema de interacciones cuidadosamente diseñado, que organiza e induce la influencia recíproca entre los integrantes de un equipo. Se desarrolla a través de un proceso gradual en el que cada miembro y todos se sienten mutuamente comprometidos con el aprendizaje de los demás, generando una interdependencia positiva que no implique competencia. El aprendizaje colaborativo se adquiere a través del empleo de métodos de trabajo grupal, caracterizado por la interacción y el aporte de todos en la construcción del conocimiento. En el aprendizaje colaborativo el trabajo grupal apunta a compartir la autoridad, a aceptar la responsabilidad y el punto de vista del otro, y a construir consenso con los demás.
"El aprendizaje colaborativo es un resultado del trabajo colaborativo" [2]. Como se ha venido exponiendo en las líneas anteriores, este último es una técnica que se centra en el razonamiento para el pensamiento divergente o pensamiento de la creatividad, a través de actividades de aprendizaje basadas en el principio de la socialización didáctica y la interdependencia positiva entre los aprendices. Esa interdependencia es el corazón de las actividades colaborativas que definen la colaboración y transforman el trabajo grupal en trabajo en equipo. Para lograr una interdependencia positiva entre los estudiantes, organizarlos en grupos y decirles que trabajen juntos puede no ser suficiente [12].

Dada su habitual confusión, cabe mencionar que el aprendizaje colaborativo se diferencia del cooperativo. En este último, se hace una división marcada de la tarea que cada miembro debe realizar, y la responsabilidad del desarrollo de construcción de saberes recae fundamentalmente en el profesor. En cambio, con el primero, el colaborativo, las metas son comunes a lo largo de todo el proceso y la autogestión de los conocimientos se hace cada vez más evidente. Como lo expone [13], "cada paradigma representa un extremo del proceso de enseñanza-aprendizaje que va de ser altamente estructurado por el profesor (cooperativo) hasta dejar la responsabilidad del aprendizaje principalmente en el estudiante (colaborativo)". Ambos tipos de aprendizaje, cooperativo y colaborativo, se fundamentan en el constructivismo. Sin embargo, el cooperativo responde a la vertiente piagetiana y el colaborativo al enfoque sociocultural [14].

Para incorporar en el aula el trabajo colaborativo como una estrategia didáctica para la enseñanza-aprendizaje, y, que como se mencionó anteriormente, redunde en un aprendizaje colaborativo, se hace necesaria la utilización de técnicas que lleven a la práctica la estrategia. 
El trabajo colaborativo como estrategia didáctica para la enseñanza/aprendizaje de la programación: una revisión sistemática de literatura

Tabla 2. Categorías de TAC. Fuente: [3].

\begin{tabular}{|c|c|c|}
\hline Categoría & Descripción & Casos Tipo \\
\hline Diálogo & $\begin{array}{l}\text { La interacción y los intercambios de los } \\
\text { estudiantes se consiguen principalmente } \\
\text { mediante la palabra hablada. }\end{array}$ & $\begin{array}{l}\text { Piensa, forma una pareja y comenta; Rueda de ideas; } \\
\text { Grupos de conversación; Para hablar, paga ficha; } \\
\text { Entrevista en tres pasos; Debates críticos. }\end{array}$ \\
\hline $\begin{array}{l}\text { Enseñanza recíproca } \\
\text { entre compañeros }\end{array}$ & $\begin{array}{l}\text { Los estudiantes se enseñan mutuamente } \\
\text { con decisión a dominar temáticas y a } \\
\text { desarrollar competencias relacionadas con } \\
\text { ellas. }\end{array}$ & $\begin{array}{l}\text { Toma de apuntes por parejas; Celdas de aprendizaje; } \\
\text { La pecera; Juego de rol; Rompecabezas; Equipos de } \\
\text { exámenes. }\end{array}$ \\
\hline Resolución de problemas & $\begin{array}{l}\text { Los estudiantes se centran en practicar } \\
\text { estrategias de resolución de problemas. }\end{array}$ & $\begin{array}{l}\text { Resolución de problemas por parejas pensando en } \\
\text { voz alta; Pasa el problema; Estudio de casos; Resolu- } \\
\text { ción estructurada de problemas; Equipos de análisis; } \\
\text { Investigación en grupo. }\end{array}$ \\
\hline $\begin{array}{l}\text { Organizadores de } \\
\text { información gráfica }\end{array}$ & $\begin{array}{l}\text { Los grupos utilizan medios visuales para } \\
\text { organizar y mostrar información. }\end{array}$ & $\begin{array}{l}\text { Agrupamiento por afinidad; Tabla de grupo; Matriz } \\
\text { de equipo; Cadenas secuenciales; Redes de palabras. }\end{array}$ \\
\hline Redacción & $\begin{array}{l}\text { Los estudiantes escriben para aprender } \\
\text { contenidos y competencias importantes. }\end{array}$ & $\begin{array}{l}\text { Diarios para el diálogo; Mesa redonda; Ensayos } \\
\text { diádicos; Corrección por el compañero; Escritura } \\
\text { colaborativa; Antologías de equipo; Seminario sobre } \\
\text { una ponencia. }\end{array}$ \\
\hline
\end{tabular}

Las Técnicas de Aprendizaje Colaborativo (TAC), más comunes y las que vayan surgiendo de la experiencia educativa, según [3], pueden organizarse en categorías que comparten aspectos fundamentales, a pesar de que las líneas divisorias no son precisas. Esta organización se muestra en la Tabla 2.

Es importante destacar que cada TAC representa un propósito o un enfoque particular. Sin embargo, no son competencia entre sí o mutuamente excluyentes, por el contrario, son complementarias. De hecho, en la práctica, una estrategia didáctica basada en trabajo colaborativo se puede componer de enfoques y recursos de varias TAC.

\section{MÉTODO}

Para desarrollar esta revisión sistemática de literatura se empleó el enfoque de investigación basada en evidencias del campo de la administración [15] y de la ingeniería de software [16]-[19]. Este enfoque ha sido ampliamente utilizado por la comunidad científica, porque permite crear conocimiento a partir de evidencias publicadas en estudios primarios. A continua- ción, se describen las etapas del método definido para esta revisión, las cuales se esquematizan en la Fig. 1.

\subsection{Planificación}

La planificación se centra en desarrollar un protocolo para la revisión sistemática. Este especifica el alcance de la revisión y las actividades que se deben ejecutar para lograr los objetivos. En este caso, el protocolo especificó las preguntas de interés, las estrategias de búsqueda, los criterios de inclusión/exclusión y de evaluación de calidad, y los procedimientos para la extracción y síntesis de datos. Las preguntas de interés que se trabajaron en esta revisión sistemática fueron las presentadas en la introducción. Los demás elementos del protocolo se describen en las siguientes subsecciones. Es importante señalar que esta revisión asumió un "enfoque centrado en el protocolo", es decir, que las estrategias de búsqueda estaban definidas antes de iniciar el proceso, contrario a un "enfoque iterativo", en donde al ejecutar las búsquedas se van modificando los criterios con el fin de incluir más documentos potenciales. 
El trabajo colaborativo como estrategia didáctica para la enseñanza/aprendizaje de la programación: una revisión sistemática de literatura

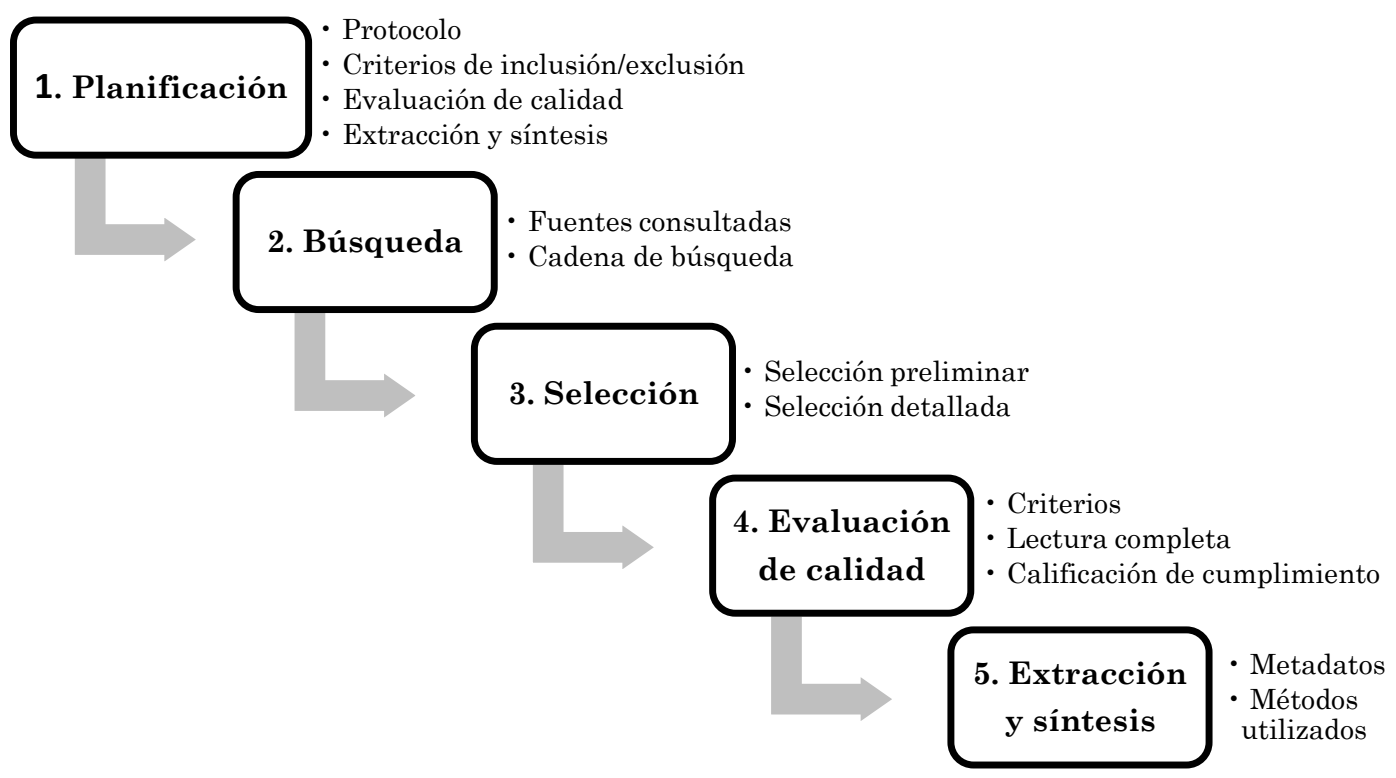

Fig. 1. Esquema del método de revisión empleado. Fuente: autores.

\subsection{Búsqueda}

Un factor fundamental para una revisión sistemática de literatura es la definición de un proceso de búsqueda exhaustivo e imparcial [18]. Como primer elemento, las fuentes utilizadas para efectuar búsquedas con palabras clave fueron las propuestas en el análisis de resultados de [20]: la base de citaciones SCOPUS, y las bases de datos de publicaciones científicas ScienceDirect, IEEE Explore Digital Library y ACM Digital Library.

El segundo elemento definido fue una cadena de búsqueda compuesta de palabras y frases clave que sirvieron para ubicar documentos potencialmente relevantes, por medio de las funciones de búsqueda disponibles en las fuentes seleccionadas. Fue construida a partir de prototipos de expresiones y operadores booleanos que fueron probados en SCOPUS. Luego, la expresión de búsqueda fue refinada con la inclusión de sinónimos, formas alternativas de escritura de los términos, y otras palabras o frases clave identificadas en los registros bibliográficos obtenidos al utilizar las expresiones de búsqueda. Estas acciones fueron ejecutadas de forma iterativa, dando como resultado la cadena de búsqueda básica compuesta por los siguientes términos clave: (teaching OR learning OR education) AND ("collaborative learning" OR "cooperative learning" OR cscl OR csgf OR "social learning" OR "group learning" OR "team learning") AND ("computer programming" OR "programming course" OR CS1).

El resultado obtenido al utilizar la cadena de búsqueda en las cuatro fuentes seleccionadas fue un conjunto de 95 registros bibliográficos de documentos científicos potencialmente relevantes, publicados entre los años 2013 y 2017 en revistas o conferencias, en idioma inglés o español, y relacionados con ciencias de la computación e ingeniería. Estos 95 registros bibliográficos fueron depurados con la eliminación de duplicados, dando como resultado 87 registros, los cuales fueron almacenados y procesados en el software MENDELEY DESKTOP [21]. 
El trabajo colaborativo como estrategia didáctica para la enseñanza/aprendizaje de la programación: una revisión sistemática de literatura

\subsection{Selección}

Los 87 registros bibliográficos potencialmente relevantes se sometieron a un proceso de selección en dos pasos. El primer paso fue una selección preliminar a partir de la revisión del título del documento y del resumen para seleccionar aquellos que hacían mención explícita a técnicas de trabajo colaborativo empleadas como estrategia didáctica en cursos de programación. Con este filtro se seleccionaron 64 registros bibliográficos, es decir, el $73,56 \%$ de los 87 registros potencialmente relevantes.

El segundo paso fue una selección detallada con base en una lectura preliminar del texto completo de los documentos referenciados en los 64 registros bibliográficos seleccionados en el primer paso. Esto implicó la recopilación de los documentos utilizando bases de datos a las cuales están suscritas la Universidad de Nariño, la Universidad Cauca y la Institución Universitaria CESMAG, y la colaboración de personas de otras instituciones académicas con acceso a fuentes de documentos científicos que no se tenían disponibles en las mencionadas instituciones. La revisión rápida del texto completo se enfocó en identificar si se hacía mención directa sobre los elementos de interés para la revisión sistemática de literatura. Con el segundo paso de la selección se obtuvieron 48 documentos, es decir, el 55,17\% de los 87 registros potencialmente relevantes.

\subsection{Evaluación de calidad}

Los documentos seleccionados fueron evaluados de acuerdo con cinco criterios para asegurar su calidad y pertinencia para la revisión sistemática: relevancia del contenido para dar respuesta a las preguntas de interés de la revisión, claridad en el objetivo de la investigación, descripción adecuada del contexto en el cual se desarrolló la investigación, claridad y rigor del diseño metodológico de la investigación, y rigor científico en el análisis de los datos. Los cinco criterios cubren tres aspectos principales de calidad: rigor, credibilidad y relevancia.

La evaluación de calidad implicó la lectura del texto completo de los 48 documentos y la calificación del cumplimiento o incumplimiento de los criterios de calidad definidos. Con esta evaluación se llegó a un conjunto de 40 documentos que sirvieron como fuentes primarias de datos para la revisión sistemática de literatura. En la Tabla 3 se presenta un resumen cuantitativo del proceso de búsqueda, selección y evaluación de calidad, y en la Tabla 4 se presentan las referencias a los 40 documentos que fueron revisados y ordenados de acuerdo con el año de publicación.

\begin{tabular}{|c|c|c|c|}
\hline Fase & $\begin{array}{c}\text { Indexado en } \\
\text { varias fuentes }\end{array}$ & $\begin{array}{l}\text { Indexado en } \\
\text { una fuente }\end{array}$ & $\begin{array}{c}\text { Total sin } \\
\text { duplicados }\end{array}$ \\
\hline Búsqueda & 7 & 80 & 87 \\
\hline Selección preliminar & 6 & 58 & 64 \\
\hline Selección detallada & 5 & 43 & 48 \\
\hline Evaluación de calidad & 4 & 36 & 40 \\
\hline
\end{tabular}

\begin{tabular}{ccc}
\multicolumn{4}{c}{ Tabla 4. Documentos revisados. Fuente: autores. } \\
\hline Año & No. & Referencias \\
\hline 2013 & 8 & {$[22]-[29]$} \\
2014 & 7 & {$[30]-[35]$} \\
2015 & 12 & {$[36]-[47]$} \\
2016 & 11 & {$[48]-[57]$} \\
2017 & 4 & {$[58]-[61]$}
\end{tabular}


El trabajo colaborativo como estrategia didáctica para la enseñanza/aprendizaje de la programación: una revisión sistemática de literatura

\subsection{Extracción de datos y síntesis de resultados}

La etapa final se enfocó en la extracción de datos relevantes para responder las preguntas de interés y en la síntesis de los resultados obtenidos. De cada documento revisado se depuraron y extrajeron los siguientes metadatos obtenidos en los registros bibliográficos: título, año de publicación, nombre de la publicación, tipo de documento, autores y país de la institución a la cual estaban adscritos los autores a la fecha de publicación del artículo. Como parte del análisis del contenido se extrajeron los métodos utilizados para desarrollar la investigación, así como una identificación preliminar del tipo de enfoque dado a los estudios, en lo referente al tipo de aprendizaje involucrado en ellos: colaborativo o cooperativo.

Para la identificación de las TAC, se empleó la clasificación general propuesta por [3], en la cual se definen también los casos tipo para cada categoría de clasificación. De esta subclasificación, se sintetizaron las denominaciones comunes de las estrategias o técnicas colaborativas presentes en los diferentes estudios. De igual manera, a estas denominaciones comunes se les sintetizaron unos propósitos generales con el fin de caracterizar cada TAC y describir sus diferentes alcances.

Se consolidaron también otros aportes importantes de los autores en indagación, que no habían sido relacionados en apartes anteriores (ver Tabla 5).

\section{RESULTADOS}

Los resultados obtenidos con la revisión de los 40 documentos seleccionados se estructuraron en cinco secciones que se presentan a continuación: aspectos generales, enfoque de aprendizaje y tipos de estudios, identificación de las TAC, síntesis de sus propósitos, y otros aportes significativos de los autores bajo indagación.

\subsection{Aspectos generales}

El primer aspecto general identificado fue la ubicación geográfica de los autores de los documentos revisados con el fin de establecer porcentualmente la procedencia de los documentos por países. El porcentaje de producción para cada país fue calculado con base en la afiliación de los autores de cada documento, siguiendo el enfoque de crédito equivalente en la autoría utilizado por [62]. En la Fig. 2 se presenta la distribución de los países de origen de los documentos revisados.

El segundo aspecto general fue la identificación del tipo de publicación revisada. La mayoría de documentos (75 \%) fueron artículos presentados y publicados en conferencias académicas. Este hecho puede juzgarse como positivo, dado que este tipo de publicaciones permiten difundir y conocer los últimos adelantos en investigación en diferentes áreas del conocimiento. Por su parte, un $25 \%$ corresponden a artículos publicados en revistas científicas indexadas lo cual también es positivo dado que este tipo de documentos son los que tienen mayor rigurosidad en la revisión por parte de editores y revisores a ciegas. A pesar de tenerse en cuenta en etapas anteriores, en los resultados finales no se obtuvieron documentos de tipo capítulo de libro.

\subsection{Enfoque de aprendizaje y tipos de estudios}

Otro aspecto a tener en cuenta en los resultados fue la identificación preliminar del enfoque dado a los estudios, en lo referente al tipo de aprendizaje involucrado en ellos. A pesar de que, como se menciona en la Sección 2, la literatura especializada establece diferencias entre el aprendizaje colaborativo y el aprendizaje cooperativo, algunos de los estudios reportados [22], [25], [27], [41], [51], [55], [60] manejan indistintamente estos conceptos. Mediante una revisión detallada se evidenció que el enfoque de estos estudios es más hacia el 
El trabajo colaborativo como estrategia didáctica para la enseñanza/aprendizaje de la programación: una revisión sistemática de literatura

aprendizaje colaborativo, objeto del presente estudio, por lo que se decidió mantenerlos como fuentes primarias de datos para la revisión sistemática.

En cuanto a la identificación de los métodos de investigación utilizados, se encontró que casi en su totalidad (39 documentos) reportan estudios primarios con componentes empíricos en diversos contextos. Solo un (1) documento reporta revisión de literatura. Para tener una mejor comprensión de las contribuciones de cada artículo de los 39 analizados, se utilizaron las cate- gorías sugeridas por [63], para analizar, clasificar y categorizar los tipos de estudios descritos en los artículos, obteniendo: 24 documentos $(61,5 \%)$ que corresponden a la categoría de Propuesta de Solución, 12 documentos $(30,8 \%)$ que corresponden a la categoría Investigación de Evaluación, y, 3 documentos $(7,7 \%)$ que corresponden a Artículos de opinión.

Se utilizaron estos enfoques y las categorías descritas para crear un gráfico de burbujas (ver Fig. 3), mostrando la distribución de los estudios en cada enfoque.

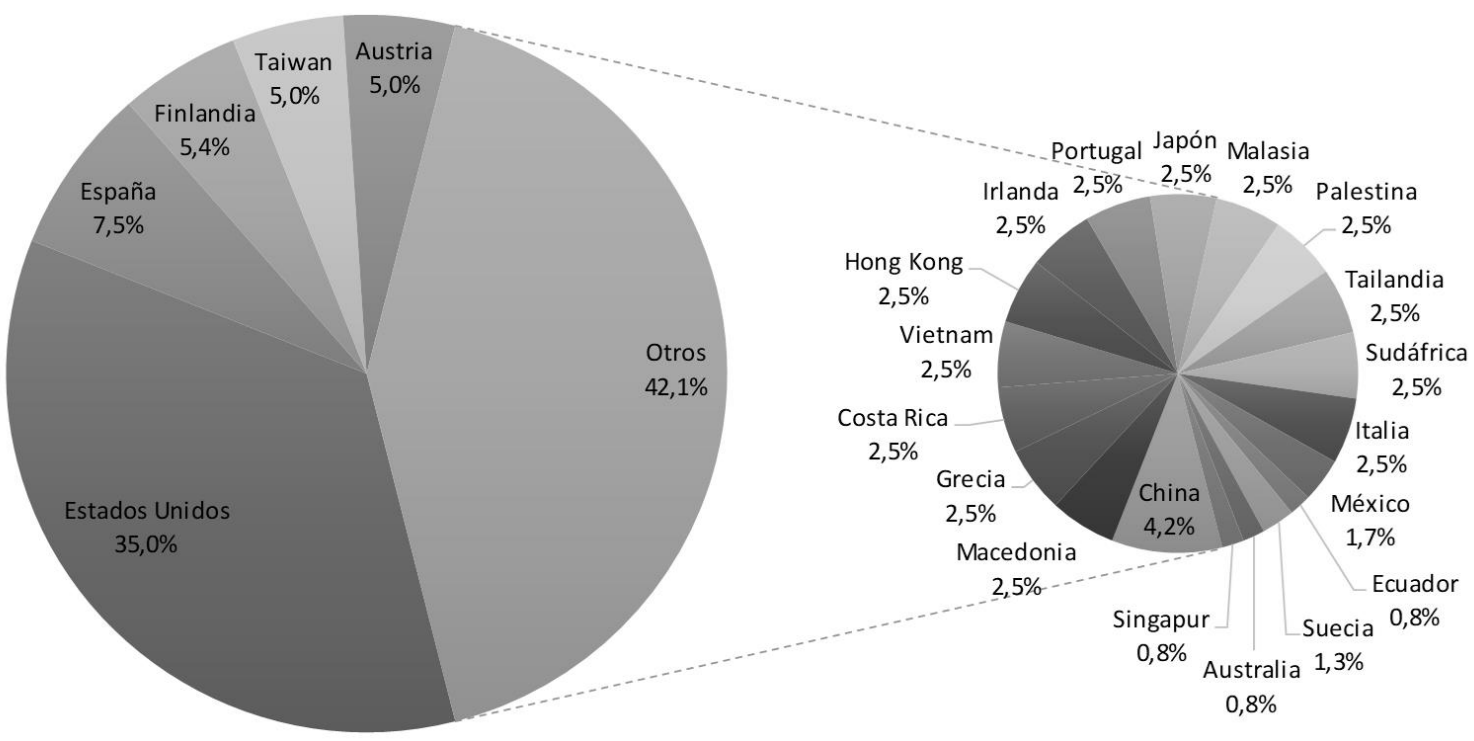

Fig. 2. Países de origen de los documentos revisados. Fuente: autores.

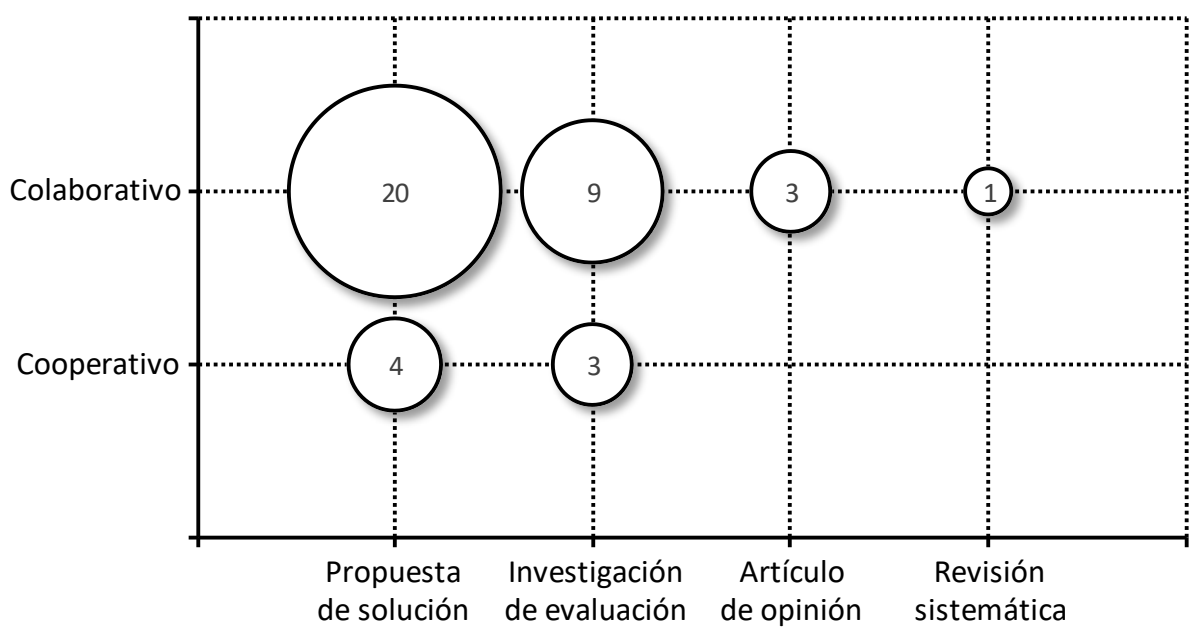

Fig. 3. Artículos por tipo de estudio (eje x) y enfoque de aprendizaje (eje y). Fuente: autores. 
El trabajo colaborativo como estrategia didáctica para la enseñanza/aprendizaje de la programación: una revisión sistemática de literatura

Tabla 5. Otros aportes significativos de los autores. Fuente: autores.

\begin{tabular}{|c|c|}
\hline Autores & Aporte \\
\hline [23] & $\begin{array}{l}\text { En la enseñanza de aquellos cursos relacionados con la programación, se suele utilizar una metodología de } \\
\text { enseñanza/aprendizaje basada en la resolución de problemas, que a veces implica la aplicación de técnicas como } \\
\text { la programación en pares. Además, para realizar tareas relacionadas con la programación, es común utilizar } \\
\text { entornos de desarrollo integrados profesionales. Por lo tanto, puede ser interesante integrar herramientas en } \\
\text { estos entornos para que puedan apoyar el proceso de enseñanza/aprendizaje. }\end{array}$ \\
\hline$[24]$ & $\begin{array}{l}\text { En los cursos de programación, a menudo se pide a los estudiantes que trabajen en grupos para escribir progra- } \\
\text { mas. Los IDE existentes, sin embargo, no fomentan las interacciones concurrentes entre los programadores } \\
\text { estudiantiles. Los estudiantes a menudo encuentran dificultades en actividades de colaboración, compartiendo } \\
\text { recursos, revisando código y discutiendo ideas, especialmente cuando el grupo no puede llegar a un acuerdo } \\
\text { común en reuniones presenciales en un momento y lugar conveniente. }\end{array}$ \\
\hline$[25]$ & $\begin{array}{l}\text { Aprender un lenguaje de programación es difícil, como los resultados académicos en diferentes contextos lo } \\
\text { evidencian. Para muchos estudiantes, incluso parece ser un obstáculo insuperable. }\end{array}$ \\
\hline$[26]$ & $\begin{array}{l}\text { El estudio de las Ciencias de la Computación se concibe necesariamente como una actividad colaborativa que se } \\
\text { centra más en la resolución de problemas y en el pensamiento algorítmico que en la programación. }\end{array}$ \\
\hline [30] & $\begin{array}{l}\text { La motivación es un factor muy importante para una instrucción exitosa. Es especialmente relevante en contex- } \\
\text { tos de aprendizaje colaborativo, donde la interacción social juega un papel importante. }\end{array}$ \\
\hline [33] & $\begin{array}{l}\text { En contextos educativos, el éxito de la programación y la persistencia en la disciplina computacional se han } \\
\text { correlacionado positivamente con el sentido de comunidad de programadores y la capacidad de comunicarse con } \\
\text { los demás. }\end{array}$ \\
\hline [36] & $\begin{array}{l}\text { Un número importante de tareas académicas debe ser resuelto en colaboración por grupos de estudiantes. Los } \\
\text { sistemas CSCL apoyan esta colaboración mediante espacios de trabajo y herramientas compartidos que permiten } \\
\text { la comunicación y la coordinación entre los estudiantes. La colaboración e interacción exitosas pueden depender } \\
\text { de los criterios seguidos al formar los grupos de estudiantes. }\end{array}$ \\
\hline [39] & $\begin{array}{l}\text { Factores relacionados con las interacciones y las discusiones entre estudiantes pueden conducir a mejores resul- } \\
\text { tados de aprendizaje en los cursos de programación. }\end{array}$ \\
\hline [40] & $\begin{array}{l}\text { El método del aula invertida se ha utilizado con diferentes enfoques y directrices, sin embargo, un único proceso } \\
\text { unificado aún no se ha descrito. }\end{array}$ \\
\hline [41] & $\begin{array}{l}\text { Los llamados "equipos ligeros" son equipos de clase en los que los miembros del equipo tienen poco o ningún } \\
\text { impacto directo en las calificaciones finales de cada uno, pero donde existe un componente significativo de la } \\
\text { enseñanza/aprendizaje entre compañeros. }\end{array}$ \\
\hline [43] & $\begin{array}{l}\text { La inclusión de la gamificación en cursos de programación se ha identificado como una técnica potencial que } \\
\text { podría maximizar la participación de los estudiantes y tener un impacto positivo en el aprendizaje. }\end{array}$ \\
\hline$[44]$ & $\begin{array}{l}\text { Un problema recurrente en el aprendizaje de la programación es que, aunque los profesores explican la gramáti- } \\
\text { ca, los estudiantes no pueden crear software por sí mismos. Inclusive, algunos estudiantes renuncian al aprendi- } \\
\text { zaje. Un enfoque de aprendizaje basado en el aula invertida y en el aprendizaje colaborativo, puede promover la } \\
\text { motivación y el entendimiento de cada estudiante hacia la programación. }\end{array}$ \\
\hline [45] & $\begin{array}{l}\text { Una forma eficaz de aprender programación de computadores es sentarse lado a lado frente al mismo computador } \\
\text { con un tutor o compañero, escribir código juntos y luego discutir lo que sucede cuando el código se ejecuta. }\end{array}$ \\
\hline [49] & $\begin{array}{l}\text { La programación de pares es un enfoque de utilidad para fomentar el pensamiento computacional en jóvenes } \\
\text { estudiantes. Sin embargo, hay muchos factores que afectan su eficacia. Entre otros, los factores sociales son a } \\
\text { menudo ignorados por los investigadores. }\end{array}$ \\
\hline [52] & $\begin{array}{l}\text { Es un tanto difícil para estudiantes de primeros años escribir un programa de computador, debido a que en su } \\
\text { gran mayoría no tienen buenas bases de Ciencias y de Matemáticas. }\end{array}$ \\
\hline [56] & $\begin{array}{l}\text { La programación en pares tiene el potencial de aumentar la confianza en los estudiantes, el disfrute del curso y } \\
\text { mejorar la tasa de finalización del mismo. Estudiantes bajo este enfoque son capaces de producir individualmente } \\
\text { un código de mejor calidad que los estudiantes bajo enfoques tradicionales. }\end{array}$ \\
\hline [58] & $\begin{array}{l}\text { La tutoría de compañeros proporciona a los estudiantes de nivel superior una oportunidad exclusiva para reforzar } \\
\text { sus conocimientos conceptuales y participar en la investigación para abordar los problemas que enfrentan los } \\
\text { estudiantes de nivel inferior. }\end{array}$ \\
\hline [59] & $\begin{array}{l}\text { La inspiración del aprendizaje del estudiante y su adecuación puede estar influenciada por varios factores am- } \\
\text { bientales que incluyen el enfoque de aprendizaje y la presión social de los compañeros de clase. }\end{array}$ \\
\hline
\end{tabular}

\subsection{Identificación de las TAC}

En todos los documentos se encontraron menciones explícitas a varias TAC, las cuales se pueden enmarcar en las categorías propuestas por [3], mencionadas anteriormente (ver Tabla 2). Como se observa en la Tabla 6, 21 documentos (52,5\%) ha- cen mención a técnicas que se agrupan en la categoría de Redacción, técnicas en las que la interacción y los intercambios de los estudiantes se consiguen principalmente mediante la palabra escrita. La mayor frecuencia en esta categoría radica en que el fin último al elaborar un programa de computador es su componente escrito, que 
El trabajo colaborativo como estrategia didáctica para la enseñanza/aprendizaje de la programación: una revisión sistemática de literatura

es el que se compila y el que a la postre "funciona". Los estudios agrupados en esta categoría presentan actividades de escritura colaborativa integrales, en las que se le da relevancia también al componente técnico (los algoritmos, la arquitectura y el código ejecutable). Un solo estudio [30], presenta una estrategia colaborativa integrada, que no se enmarca dentro de las categorías propuestas por [3], la cual se describe más adelante en la Sección 4.4.

$\mathrm{Al}$ identificar los casos tipo en cada una de las categorías (de acuerdo con lo que se presenta en la Tabla 2), algunos fueron más frecuentes que otros. Como se observa en la Tabla 7, los tres casos con mayor frecuencia fueron "Escritura colaborativa" con 18 ocurrencias, "Celdas de aprendizaje" con 7 ocurrencias, y "Corrección por el compañero" y "Resolución estructurada de problemas", con 3 ocurrencias.
En la identificación de los casos tipo, se tuvo en cuenta la esencia de la técnica, como lo mencionan [3], ya que cada uno de estos casos puede describir de forma genérica múltiples estrategias colaborativas, pero con denominaciones diferentes. Por lo tanto, se realizó una síntesis en un conjunto más pequeño de denominaciones, a manera de subcategorías de TAC, que reunieran los elementos comunes de las estrategias encontradas en los documentos. El resultado de este análisis es el que se muestra en la Tabla 8. En el Tabla 9 se presentan los casos tipo y la denominación común de la estrategia o técnica colaborativa asociada, así como su correspondiente número de ocurrencias.

En la Tabla 8, se resumen cuantitativamente los once casos tipo identificados, ordenados de acuerdo con el porcentaje de documentos en donde se encontraron técnicas relacionadas, y las referencias bibliográficas correspondientes.

Tabla 6. TAC mencionadas en documentos organizadas por categorías.

\begin{tabular}{lcr}
\multicolumn{3}{c}{ Fuente: autores. } \\
\hline Categoría & No. & \multicolumn{1}{c}{$\%$} \\
\hline Diálogo & 1 & 2,5 \\
Enseñanza recíproca entre compañeros & 11 & 27,5 \\
Resolución de problemas & 3 & 7,5 \\
Organizadores de información gráfica & 3 & 7,5 \\
Redacción & 21 & 52,5 \\
Otra & 1 & 2,5 \\
\hline
\end{tabular}

\begin{tabular}{llc}
\multicolumn{2}{l}{ Tabla 7. TAC mencionadas en documentos organizadas por casos tipo. Fuente: autores. } \\
\hline Categoría & Caso Tipo & F \\
\hline Diálogo & Grupos de conversación & 1 \\
\hline \multirow{2}{*}{ Enseñanza recíproca entre compañeros } & Celdas de aprendizaje & 7 \\
& Juego de roles & 2 \\
& Gamificación & 2 \\
\hline \multirow{2}{*}{ Resolución de problemas } & Resolución estructurada de problemas & 3 \\
\hline \multirow{2}{*}{ Organizadores de información gráfica } & Mundos virtuales & 1 \\
& Entorno virtual & 1 \\
& Herramienta específica & 1 \\
\hline \multirow{2}{*}{ Redacción } & Escritura colaborativa & 18 \\
& Corrección por el compañero & 3 \\
\hline Otra & Estrategia colaborativa integrada & 1 \\
\hline
\end{tabular}


El trabajo colaborativo como estrategia didáctica para la enseñanza/aprendizaje de la programación: una revisión sistemática de literatura

Tabla 8. Resumen cuantitativo de la identificación de TAC. Fuente: autores.

\begin{tabular}{|c|c|c|c|c|}
\hline Caso tipo & $\begin{array}{c}\text { No. de } \\
\text { TAC }\end{array}$ & $\begin{array}{c}\text { No. de } \\
\text { documentos }\end{array}$ & $\begin{array}{c}\text { \% de } \\
\text { documentos }\end{array}$ & Referencias Bibliográficas \\
\hline Escritura colaborativa & 4 & 18 & 45,0 & $\begin{array}{l}{[23]-[25],[27],[29],[33],[36],} \\
{[37],[42],[45],[47]-[50],[52],} \\
{[53],[56],[61]}\end{array}$ \\
\hline Celdas de aprendizaje & 3 & 7 & 17,5 & $\begin{array}{l}{[26],[35],[40],[41],[44],[58],} \\
{[60]}\end{array}$ \\
\hline Corrección por el compañero & 1 & 3 & 7,5 & {$[28],[46],[55]$} \\
\hline Resolución estructurada de problemas & 3 & 3 & 7,5 & [32], [38], [59] \\
\hline Juego de roles & 1 & 2 & 5,0 & {$[22],[51]$} \\
\hline Gamificación & 2 & 2 & 5,0 & {$[31],[43]$} \\
\hline Mundos virtuales & 1 & 1 & 2,5 & [34] \\
\hline Entorno virtual & 1 & 1 & 2,5 & [57] \\
\hline Herramienta específica & 1 & 1 & 2,5 & {$[54]$} \\
\hline Grupos de conversación & 1 & 1 & 2,5 & {$[39]$} \\
\hline Otra & 1 & 1 & 2,5 & [30] \\
\hline
\end{tabular}

Tabla 9. Síntesis de las denominaciones comunes de TAC. Fuente: autores.

\begin{tabular}{ll}
\hline \multicolumn{1}{c}{$\begin{array}{c}\text { Caso Tipo } \\
\text { Escritura colaborativa }\end{array}$} & Denominaciones comunes identificadas en los documentos \\
\hline Celdas de aprendizaje & $\begin{array}{l}\text { GitHub (2), IDE Colaborativo (6), Programación en pares (9), Wiki } \\
(1)\end{array}$ \\
\hline Corrección por el compañero & $\begin{array}{l}\text { Tutoría de compañeros (2), Pares Adversarios Colaborativos (1), } \\
\text { Aula invertida (4) }\end{array}$ \\
\hline Resolución estructurada de problemas & Evaluación de código por pares (3) \\
\hline Juego de roles & $\begin{array}{l}\text { Aprendizaje basado en problemas y proyectos (1), Ingeniería de } \\
\text { Software basada en componentes (1), Hackathon (1) }\end{array}$ \\
\hline Gamificación & \begin{tabular}{l} 
Juego de roles (2) \\
\hline Mundos virtuales
\end{tabular} \\
\hline Entorno virtual & $\begin{array}{l}\text { Sistema de discusión en línea gamificado (1), Actividad de aprendi- } \\
\text { zaje gamificada (1) }\end{array}$ \\
\hline Herramienta específica & $\begin{array}{l}\text { MeLoISE - Meaningful Logical Interpretations of Simulated Envi- } \\
\text { ronments (1) }\end{array}$ \\
\hline Grupos de conversación & Entorno virtual colaborativo inteligente (1) \\
\hline Otra & Plataforma como un servicio (PaaS - Platform as a Service) (1) \\
\hline
\end{tabular}

\subsection{Síntesis de los propósitos de las TAC}

La síntesis de los propósitos de las denominaciones comunes de TAC identificadas en los documentos se realizó con el fin de describir su alcance. Para lograr esto, se revisaron las descripciones de las TAC en los 40 documentos y se identificaron elementos clave, los que permitieron la síntesis que se presenta en la Tabla 10. 
El trabajo colaborativo como estrategia didáctica para la enseñanza/aprendizaje de la programación: una revisión sistemática de literatura

Tabla 10. Propósitos de las denominaciones comunes de TAC. Fuente: Autores.

TAC

Propósito

Git-Hub

Es un repositorio de software en línea que permite la administración de código fuente [53]. [37] lo presentan con un enfoque de programación colaborativa en línea, denominada también programación o codificación social.

Es un IDE que integra características colaborativas para crear un entorno interactivo y sensible, en el que la orientación del trabajo en tiempo real, la comunicación y la colaboración pueden ser liberadas [24]. Se IDE Colaborati- presenta también bajo la denominación de "Instrumento de Asistencia para la Programación", el cual vo utiliza el concepto de aprendizaje colaborativo y el aprendizaje basado en la investigación, para estimular el aprendizaje de los estudiantes haciendo énfasis en el trabajo grupal [52]. Ejemplos de estos entornos: COLLECE [36], CODECHELLA [45], COLE-PROGRAMMING [23] y OSBIDE [33].

Es una técnica colaborativa en la que dos estudiantes trabajan para resolver un problema común de programación. El estudiante en el papel de "conductor" escribe el código, controlando el teclado y el ratón; el otro estudiante, el "navegador", revisa el código cuando el conductor lo escribe, haciendo sugerencias, señalando errores y haciendo preguntas [42], [61]. Permite a los estudiantes producir programas de Programación en computador de mayor calidad, en menos tiempo y con mejor comprensión y disfrute [48]. Los estudiantes pares tienen la posibilidad de aprender activamente, al descubrir nuevos temas o al explicarlos a otros [27]. Se introdujo originalmente como parte de la programación extrema [56]. Su enfoque colaborativo, fomenta el desarrollo del pensamiento computacional [49]. En [29], los autores presentan una combinación de la programación en pares y la enseñanza en pares, las que producen un mejor ambiente de aprendizaje para estudiantes principiantes, que cuando una o las dos no se utilizan.

Se presenta un CSCL - Computer-Supported Collaborative Learning en línea basado en Wiki [50], enfocado a resolver ejercicios de programación, que, dada su filosofía doblemente colaborativa, permite a los estudiantes realizar tres acciones clave: ver, editar y comentar las páginas de wiki.

Es una actividad colaborativa puntualmente diseñada para brindar retroalimentación a los compañeros. Puede ser vista como una estrategia de aprendizaje que ayuda al estudiante a conocer las fortalezas y debilidades de sus pares, a plantear observaciones a objetivos no alcanzados, y, a desarrollar y mejorar su capacidad metacognitiva, su capacidad de pensamiento crítico, su rendimiento en pruebas y sus habilidades profesionales [55]. [28] presentan un enfoque de aprendizaje activo inspirado en el proceso de inspec-

Evaluación de código por pares ción de código utilizado en la industria del software, bajo la denominación de Revisión Pedagógica de Código; la definen como una actividad colaborativa en la que un pequeño equipo de estudiantes liderado por un moderador capacitado: (a) explora las soluciones de programación de otros, (b) contrasta el código con una lista de las mejores prácticas de codificación, y (c) discute y registra las cuestiones que surjan. Por su parte, en [46], los autores plantean una detección colaborativa de código fuente similar, comprobando anónimamente el código y brindando la retroalimentación correspondiente.

Es un mundo virtual diseñado para apoyar el aprendizaje de la Programación Lógica, que permite a los MeLoISE estudiantes experimentar bajo una interfaz visual colaborativa con el lenguaje de programación Prolog [34].

El aprendizaje basado en problemas y el aprendizaje orientado por proyectos son dos métodos inductivos Aprendizaje de aprendizaje ampliamente utilizados. El primero tiene lugar cuando los estudiantes son enfrentados a basado en un problema auténtico como punto de partida al aprendizaje, y en el segundo, el proceso de aprendizaje se problemas y proyectos estructura alrededor de un proyecto. [32] presentan una propuesta que enmarca estos tipos de aprendizaje con énfasis especial en tres ejes fundamentales: (1) promover la autonomía de los estudiantes; (2) fomentar el aprendizaje colaborativo; y (3) generar procesos de evaluación participativos.

Es una rama de la ingeniería de software que busca reutilizar piezas de código pre-elaborado que permiten

Ingeniería de realizar diversas tareas, conllevando a diversos beneficios como las mejoras de calidad, la reducción del Software basada ciclo de desarrollo y un mayor retorno sobre la inversión. [38] la presenta con un enfoque colaborativo, en en componentes el que diferentes equipos de estudiantes desarrollan partes de un software, cuyos diseños son probados por otros equipos en términos de interoperabilidad y extensibilidad de componentes.

Es un evento donde los programadores de computadores colaboran para construir nuevas soluciones de software en una cantidad limitada de tiempo. Su objetivo principal es tener un producto mínimo viable al

Hackathon final del evento. Este enfoque puede ayudar a los estudiantes a solidificar sus habilidades en la resolución de problemas, sus habilidades de programación, sus habilidades de comunicación y sus resultados académicos. Permite a los estudiantes aprender, colaborar y crear soluciones innovadoras, mientras que ganan experiencia trabajando en un entorno de equipo [59].

Se concibe como la integración de los Entornos Virtuales Colaborativos y la Inteligencia Artificial. Es un Entorno virtual sistema computacional diseñado como un espacio conceptual para que un usuario, en condiciones espacio colaborativo temporales distintas, interactúe con otros usuarios o con elementos del entorno para construir su aprendiinteligente zaje. El componente inteligente es un elemento del entorno provisto de una base de conocimientos y de una estrategia pedagógica basada en la formulación de consultas por parte del aprendiz [57].

Es una forma de mentoría que suele tener lugar entre una persona que ha vivido una experiencia específica (mentor de compañeros) y una persona que es nueva en esa experiencia (el compañero aprendiz). Este

Tutoría de compañeros modelo de colaboración entre compañeros promueve un nuevo tipo de aprendizaje orientado al servicio, que inspira innovación y conduce a la búsqueda de maneras de superar limitaciones conceptuales comunes [58]. 
El trabajo colaborativo como estrategia didáctica para la enseñanza/aprendizaje de la programación: una revisión sistemática de literatura

TAC

Propósito

\begin{tabular}{|c|c|}
\hline $\begin{array}{l}\text { Pares adversa- } \\
\text { rios colaborati- } \\
\text { vos }\end{array}$ & $\begin{array}{l}\text { Es un proceso de desarrollo de software en el que los desarrolladores trabajan juntos de manera colabora- } \\
\text { tiva en identificar cómo diseñar y estructurar el problema, aclaran los requisitos y discuten la estrategia; } \\
\text { se mueven en papeles adversarios. A un desarrollador se le asigna la responsabilidad de implementar el } \\
\text { diseño y al otro se le asigna la tarea de escribir casos de prueba de caja negra para los diversos componen- } \\
\text { tes del problema. El objetivo del implementador es crear código "inquebrantable" y el del probador es } \\
\text { "romper" el código [35]. }\end{array}$ \\
\hline Aula invertida & $\begin{array}{l}\text { Es un método de enseñanza en el que la teoría se estudia en casa y los ejercicios se realizan en el aula. } \\
\text { Esto da lugar a una filosofía en la que el tiempo de contacto de los profesores y los estudiantes está desti- } \\
\text { nado a ser utilizado de la manera más eficaz posible. Ayudados por la tecnología, los estudiantes pueden } \\
\text { estudiar el material básico necesario de manera independiente, permitiendo abordar temas más avanza- } \\
\text { dos, el aprendizaje entre compañeros y la tutoría por pares en las sesiones de ejercicios [40]. El aula } \\
\text { invertida induce a los estudiantes a explorar el conocimiento a través de la investigación y la colaboración. } \\
\text { Por definición, derrumba los papeles tradicionales del profesor y del estudiante, y mueve las actividades } \\
\text { "en clase" fuera del aula [60]. La característica principal de este enfoque aplicado a la programación, es } \\
\text { pasar la mayor parte del tiempo de una clase en actividades de colaboración entre los estudiantes, con el } \\
\text { fin de hacer del desarrollo de software algo práctico [44]. }\end{array}$ \\
\hline Juego de roles & $\begin{array}{l}\text { Es una situación creada en la que los estudiantes representan o asumen deliberadamente personalidades o } \\
\text { identidades que, normalmente no admitirían, para alcanzar determinados objetivos de aprendizaje. Se } \\
\text { presenta un enfoque específico de esta técnica orientado a disciplinas de las ciencias, la tecnología, la } \\
\text { ingeniería y las matemáticas denominado POGIL - Process-Oriented Guided Inquiry Learning. POGIL es } \\
\text { una estrategia centrada en el estudiante; los estudiantes trabajan en pequeños grupos con roles individua- } \\
\text { les para asegurar que todos estén completamente involucrados en el proceso de aprendizaje [22], [51]. }\end{array}$ \\
\hline $\begin{array}{l}\text { Sistema de } \\
\text { discusión en } \\
\text { línea gamific }\end{array}$ & $\begin{array}{l}\text { Es el componente de discusión de un CSCL al que se le han agregado elementos gamificados. Su propósito } \\
\text { es aumentar la colaboración entre los estudiantes, el nivel de compromiso, y la eficiencia de la comunica- } \\
\text { ción al interior del curso, reduciendo los tiempos de respuesta asincrónica [31]. }\end{array}$ \\
\hline $\begin{array}{l}\text { Actividad de } \\
\text { aprendizaje } \\
\text { gamificada }\end{array}$ & $\begin{array}{l}\text { Es una actividad a la que se le han incorporado elementos de juego como tablas de líderes, insignias, } \\
\text { desafíos y elementos de socialización para integrar las interacciones entre instructor-persona, instructor- } \\
\text { grupo y persona-grupo. Se ocupa de la participación de los estudiantes y del desarrollo de habilidades } \\
\text { durante el proceso de aprendizaje, pero no considera el logro de los estudiantes [43]. }\end{array}$ \\
\hline $\begin{array}{l}\text { Plataforma como } \\
\text { un servicio }\end{array}$ & $\begin{array}{l}\text { Es un paradigma de producción de software. La adopción de PaaS permite el uso de máquinas virtuales } \\
\text { remotas en lugar de hardware y software local, evitando así el consumo de tiempo e instalaciones costosas, } \\
\text { así como las molestas tareas de mantenimiento. Con su aplicación, los estudiantes están capacitados para } \\
\text { cooperar, desarrollar, mantener y administrar cualquier proyecto con cualquier equipo en cualquier } \\
\text { momento sin necesidad de trasladarse a ningún laboratorio físico [54]. }\end{array}$ \\
\hline MOOC & $\begin{array}{l}\text { Es una forma de ofrecer cursos en línea. Entre otras prestaciones, proporcionan foros de discusión para } \\
\text { que los estudiantes hagan preguntas y discutan temas relacionados con el curso. El razonamiento pedagó- } \\
\text { gico subyacente de los foros de discusión se relaciona con la idea de que la interacción social fomenta la } \\
\text { construcción del conocimiento. Se habla entonces de una interacción pedagógicamente efectiva [39]. }\end{array}$ \\
\hline $\begin{array}{l}\text { Estrategia } \\
\text { colaborativa } \\
\text { integrada }\end{array}$ & $\begin{array}{l}\text { Serrano-Camara et al. [30] presentan una estrategia de aprendizaje basada en cuatro enfoques pedagógi- } \\
\text { cos: la enseñanza tradicional, el aprendizaje colaborativo, el aprendizaje colaborativo guiado por CIF } \\
\text { (Collaborative Instruction Framework), y el aprendizaje colaborativo guiado por CIF y apoyado por Mo- } \\
\text { CAS (Mobile Collaborative Argument Support, herramienta destinada a apoyar actividades de colabora- } \\
\text { ción definidas mediante CIF). Considera las cuatro dimensiones de la motivación según la teoría de la } \\
\text { autodeterminación. }\end{array}$ \\
\hline
\end{tabular}

\subsection{Otros aportes significativos de los Autores}

En la Tabla 5, se presentan otros aportes significativos de los autores bajo indagación, no relacionados en apartes anteriores.

\section{DISCUSIÓN}

En relación con los aspectos generales se encontró que la mayor parte de los estudios provienen de instituciones educativas de Estados Unidos, destacándose también estudios provenientes de instituciones de España, Finlandia, Taiwán y Austria. De otra parte, se encontró que la mayoría de documentos son artículos presentados y publicados en conferencias académicas, hecho puede que puede valorarse como positivo, dado que este tipo de publicaciones permiten difundir y conocer los últimos adelantos en investigación en diferentes áreas del conocimiento.

Por otra parte, la respuesta a la pregunta sobre los enfoques de aprendizaje y los tipos de contribuciones de los estudios 
El trabajo colaborativo como estrategia didáctica para la enseñanza/aprendizaje de la programación: una revisión sistemática de literatura

en indagación, mostró que, si bien la literatura especializada establece diferencias entre el aprendizaje colaborativo y el aprendizaje cooperativo, algunos de los estudios reportados manejan indistintamente estos enfoques, pero en una revisión más detallada se encontró que estos estudios se orientan más hacia el enfoque colaborativo, objeto del presente estudio, por lo que se decidió mantenerlos como fuentes primarias para la revisión. Para los dos enfoques se observó que la mayor parte de los estudios se concentran en Propuestas de Solución e Investigaciones de Evaluación, categorías estas sugeridas por [63] para analizar, clasificar y categorizar los tipos de estudios descritos en la literatura.

En cuanto a las TAC, definidas en la literatura científica revisada, se encontró que estas se pueden enmarcar de forma general en las categorías propuestas por [3]. La mayor parte de los estudios los agrupa la categoría de Redacción, dado que el fin último al elaborar un programa de computador es su componente escrito, que es el que se compila y el que a la postre "funciona". Por su parte, en cada categoría se identificaron unos casos tipo, teniendo en cuenta la esencia de la técnica, ya que cada uno de estos casos puede describir de forma genérica múltiples estrategias colaborativas, pero con denominaciones diferentes. Se realizó una síntesis en un conjunto más pequeño de denominaciones, a manera de subcategorías de TAC, que reúnen los elementos comunes de las estrategias encontradas en los documentos. Los tres casos con mayores frecuencias fueron escritura colaborativa, celdas de aprendizaje, corrección por el compañero y resolución estructurada de problemas.

A partir de estas subcategorías, se sintetizaron las denominaciones más comunes de las estrategias o técnicas colaborativas específicas identificadas en los documentos, describiendo brevemente sus alcances con base en los aportes de los diferentes autores, destacándose estrategias como la programación en pares y la utilización de IDE's colaborativos.

Finalmente, se extractaron otros aportes importantes de los autores en indagación, los cuales complementan y reafirman la idea común detectada a lo largo del estudio, de que la utilización de estrategias o técnicas colaborativas como apoyo en el aprendizaje de la programación, ofrece escenarios de aprendizaje alternativos, en donde el objetivo primordial es la construcción colectiva del conocimiento, que para el caso, redunde en una reducción de la ya reconocida complejidad que el área de conocimiento presenta.

\section{CONCLUSIONES}

El trabajo colaborativo empleado como una estrategia didáctica de enseñanza/aprendizaje es un tema de investigación importante en el ámbito educativo $\mathrm{y}$ computacional, debido a su posible aplicación para aumentar los beneficios de aprendizaje especialmente en estudiantes de áreas de conocimiento técnico como las ciencias de la computación, y específicamente en cursos relacionados con la programación de computadores. Debido a la habitual complejidad en la enseñanza/aprendizaje de la Programación, se han planteado diferentes enfoques didácticos que incorporan elementos de colaboración, y en ocasiones en combinación con otros enfoques, buscando consolidar estrategias que aporten posibles soluciones al problema. En este trabajo se utilizó el método de revisión sistemática (ver Sección 3) para recopilar, analizar y resumir los logros de investigación sobre el tema. Se inicia con el análisis de 95 artículos y, después de una cuidadosa inspección, se descartan 55 artículos que no cumplían con los criterios de inclusión y exclusión definidos. Los 40 trabajos restantes, fueron los que produjeron las evidencias para responder a las cuatro (4) preguntas de interés presentadas en la introducción. 
El trabajo colaborativo como estrategia didáctica para la enseñanza/aprendizaje de la programación: una revisión sistemática de literatura

De acuerdo con los resultados expuestos en la Sección 4, el colaborativo y el cooperativo son los enfoques de aprendizaje presentes en los estudios, los cuales se concentran mayoritariamente en Propuestas de Solución e Investigaciones de Evaluación (ver Fig. 3); esto respondiendo a RQ1. Se identificaron seis (6) categorías y once (11) casos tipo o subcategorías relacionadas con las Técnicas de Aprendizaje Colaborativo - TAC, definidas en la literatura científica revisada (ver Tabla 8); lo que responde a RQ2. Se sintetizaron 19 denominaciones comunes de estrategias encontradas en los documentos, es decir, las estrategias o técnicas colaborativas asociadas a cada estudio (ver Tabla 9); respondiendo a RQ3. La revisión también arrojó una cantidad importante de aportes de la comunidad investigadora que sientan una buena base para trabajos futuros, dejando en claro que el trabajo colaborativo se consolida cada vez más como una estrategia didáctica valida y pertinente, no solo para la enseñanza/aprendizaje de la Programación, sino también para otras áreas del conocimiento incluyendo las ciencias de la computación (ver Tabla 9 y 10); lo que da respuesta a RQ3 y RQ4.

De otra parte, se logró identificar un conjunto de estrategias o técnicas colaborativas orientadas específicamente a apoyar el aprendizaje de la programación. Estas son: Git-Hub, los IDE's colaborativos, la programación en pares, la evaluación de código por pares, elementos de la ingeniería de software basada en componentes, y los eventos tipo "hackathon". Asimismo, se identificaron estrategias o técnicas colaborativas de enfoque más común, que perfectamente pueden ser empleadas en otras áreas de las ciencias de la computación y del conocimiento en general. Estas son: los Wiki, el aprendizaje basado en problemas y proyectos, los entornos virtuales colaborativos inteligentes, la tutoría de compañeros, los pares adversarios colaborativos, el aula invertida, los juegos de roles, los sistemas de discusión en línea gamificados, las actividades de aprendizaje gamificadas, y los MOOC, entre otras.

Es importante destacar que cada una de las TAC identificadas representa un propósito o un enfoque particular. Sin embargo, no son competencia entre sí o mutuamente excluyentes, por el contrario, son complementarias. De hecho, en la práctica, una estrategia didáctica basada en trabajo colaborativo se puede componer de enfoques y recursos de varias TAC.

A través del desarrollo de esta revisión sistemática, es posible brindar a la comunidad académica una visión general de la investigación sobre el uso del trabajo colaborativo como recurso didáctico para la enseñanza/aprendizaje de la Programación de Computadores. Además de mostrar el creciente número de publicaciones y una variedad de enfoques para tratar el tema, también identificamos un número importante de aportes por parte de los investigadores. Esto, como se mencionó anteriormente, puede explotarse para abrir nuevas e importantes oportunidades para la investigación futura.

\section{AGRADECIMIENTOS}

Los autores expresan sus agradecimientos a sus respectivos grupos de investigación por permitir y apoyar el desarrollo de este trabajo: Grupo de Investigación Galeras.NET del Departamento de Sistemas de la Universidad de Nariño, el Grupo de Investigación y Desarrollo en Ingeniería del Software - IDIS de la Facultad de Ingeniería Electrónica y Telecomunicaciones de la Universidad del Cauca, y el Grupo de Investigación Tecnofilia de la Facultad de Ingeniería de la Institución Universitaria CESMAG.

\section{REFERENCIAS}

[1] M. Guitert and F. Jiménez, "Aprender a colaborar," in Cooperar en clase: Ideas e 
El trabajo colaborativo como estrategia didáctica para la enseñanza/aprendizaje de la programación: una revisión sistemática de literatura

instrumentos para trabajar en el aula, A. Campiglio and R. Rizzi, Eds. Madrid: M.C.E.P, 2000.

[2] J. M. Chaljub, "Trabajo colaborativo como estrategia de enseñanza en la universidad," Cuad. Pedagog. Univ., vol. 11, no. 22, pp. 6471, 2014.

[3] E. Barkley, K. P. Cross, and C. Major, Técnicas de aprendizaje colaborativo: manual para el profesorado universitario. Morata, 2007.

[4] Y. Dimitriadis, J. I. Asensio, E. Gómez, A. Martínez, M. L. Bote, G. V. Gorgojo, and L. M. Vaquero, "Middleware para CSCL: Marco de Componentes Software y Apoyo de Tecnología Grid," Rev. Iberoam. Intel. Artif., vol. 8, no. 24, pp. 21-31, 2004.

[5] M. Maldonado, "El trabajo colaborativo en el aula universitaria," Laurus, vol. 13, no. 23, pp. 263-278, 2007.

[6] T. Panitz and P. Panitz, "Encouraging the Use of Collaborative Learning in Higher Education," in University Teaching: International Perspectives, J. J. F. Forest, Ed. Taylor and Francis, 2014, pp. 161-201.

[7] B. Gros, El Ordenador invisible: hacia la apropiación del ordenador en la enseñanza, vol. 1. Barcelona, España: Gedisa Editorial, 2000.

[8] J. Salinas, "El aprendizaje colaborativo con los nuevos canales de comunicación," in Nuevas tecnologías aplicadas a la educación, J. Cabero Almenara, Ed. Síntesis, 2000, pp. 199-227.

[9] M. M. Lucero, "Entre el trabajo colaborativo y el aprendizaje colaborativo," Rev. Iberoam. Educ., pp. 1-20, 2003.

[10] C. Echazarreta, F. Prados, J. Poch, and J. Soler, "La competencia 'El trabajo colaborativo': Una oportunidad para incorporar las TIC en la didáctica universitaria. Descripción de la experiencia con la plataforma ACME (UdG)," Rev. sobre la Soc. del Conoc., no. 8, pp. 1-11, 2009.

[11] D. W. Johnson, R. T. Johnson, and E. Johnson, Los nuevos círculos de aprendizaje. La cooperación en el aula y la escuela. Aique, 1999.

[12] C. A. Collazos, L. A. Guerrero, J. A. Pino, and S. F. Ochoa, "Collaborative Scenarios to Promote Positive Interdependence among Group Members," in Groupware: Design, Implementation, and Use, Springer, Berlin, Heidelberg, 2003, pp. 356-370.

[13] L. M. Zañartu, "Aprendizaje colaborativo: una nueva forma de Diálogo Interpersonal y en Red," Rev. Digit. Educ. y nuevas Tecnol., no. 28, pp. 1-12, 2003.

[14] E. Escolano Pérez, L. T. Aragonés, and M. L. Herrero, "Percepción del alumnado universitario sobre su primera experiencia de aprendizaje colaborativo," in $V$ Congreso Mundial de Estilos de Aprendizaje, 2012, pp. $1-9$.

[15] D. Tranfield, D. Denyer, and P. Smart, "Towards a Methodology for Developing Evidence-Informed Management Knowledge by Means of Systematic Review," $\mathrm{Br}$. J. Manag., vol. 14, no. 3, pp. 207-222, Sep. 2003.

[16] B. Kitchenham, O. Pearl Brereton, D. Budgen, M. Turner, J. Bailey, and S. Linkman, "Systematic literature reviews in software engineering - A systematic literature review," Inf. Softw. Technol., vol. 51, no. 1, pp. 7-15, Jan. 2009

[17] B. A. A. Kitchenham, T. Dyba, and M. Jorgensen, "Evidence-based software engineering," 26th Int. Conf. Softw. Eng., pp. 273-281, 2004.

[18] B. Kitchenham and S. Charters, "Guidelines for performing Systematic Literature reviews in Software Engineering Version 2.3," UK: Keele University and University of Durham, 2007.

[19] B. Kitchenham, R. Pretorius, D. Budgen, O. Pearl Brereton, M. Turner, M. Niazi, and S. Linkman, "Systematic literature reviews in software engineering - A tertiary study," Inf. Softw. Technol., vol. 52, no. 8, pp. 792-805, Aug. 2010.

[20] T. Dyba, T. Dingsoyr, and G. K. Hanssen, "Applying Systematic Reviews to Diverse Study Types: An Experience Report," in First International Symposium on Empirical Software Engineering and Measurement (ESEM 2007), 2007, pp. 225-234.

[21] The Mendeley Support Team and T. M. S. Team, "Getting Started with Mendeley BT Mendeley Desktop," Mendeley Desktop. pp. 1-16, 2011.

[22] L. Beck and A. Chizhik, "Cooperative learning instructional methods for CS1: Design, implementation, and evaluation," ACM Trans. Comput. Educ., vol. 13, no. 3, pp. 1-21, 2013.

[23] F. Jurado, A. I. Molina, M. A. Redondo, and M. Ortega, "Cole-Programming: Shaping Collaborative Learning Support in Eclipse," IEEE Rev. Iberoam. Tecnol. del Aprendiz., vol. 8, no. 4, pp. 153-162, Nov. 2013.

[24] H. T. Tran, H. H. Dang, K. N. Do, T. D. Tran, and Vu Nguyen, "An interactive Web-based IDE towards teaching and learning in programming courses," in Proceedings of 2013 IEEE International Conference on Teaching, Assessment and Learning for Engineering (TALE), 2013, pp. 439-444.

[25] B. Sabitzer and S. Strutzmann, "Brain-based Programming," in 2013 IEEE Frontiers in 
El trabajo colaborativo como estrategia didáctica para la enseñanza/aprendizaje de la programación: una revisión sistemática de literatura

Education Conference (FIE), 2013, pp. 11631169.

[26] R. M. Powell, C. Murphy, A. Cannon, J. Gordon, and A. Ramachandran, "Evaluation of the emerging scholars program at Columbia University (abstract only)," in Proceeding of the 44th ACM technical symposium on Computer science education SIGCSE' '13, 2013, p. 731.

[27] B. Sabitzer, S. Pasterk, and S. Elsenbaumer, "Informatics is COOL," in Proceedings of the 8th Workshop in Primary and Secondary Computing Education on - WiPSE '13, 2013, pp. 91-94.

[28] C. D. Hundhausen, A. Agrawal, and P. Agarwal, "Talking about code," ACM Trans. Comput. Educ., vol. 13, no. 3, pp. 1-28, Aug. 2013.

[29] N. Anderson and T. Gegg-Harrison, "Learning computer science in the "comfort zone of proximal development," in Proceeding of the 44th ACM technical symposium on Computer science education SIGCSE' '13, 2013, pp. 495-500.

[30] L. M. Serrano-Cámara, M. Paredes-Velasco, C.-M. Alcover, and J. Á. Velazquez-Iturbide, "An evaluation of students' motivation in computer-supported collaborative learning of programming concepts," Comput. Human Behav., vol. 31, no. 1, pp. 499-508, Feb. 2014.

[31] A. Knutas, J. Ikonen, U. Nikula, and J. Porras, "Increasing collaborative communications in a programming course with gamification," in Proceedings of the 15th International Conference on Computer Systems and Technologies - CompSysTech '14, 2014, pp. 370-377.

[32] S. M. Rivera, M. C. Chotto, and G. A. Salazar, "A proposal for implementing PBL in programming courses," in 2014 XL Latin American Computing Conference (CLEI), 2014, pp. 1-11.

[33] C. D. Hundhausen and A. S. Carter, "Supporting Social Interactions and Awareness in Educational Programming Environments," in Proceedings of the 5th Workshop on Evaluation and Usability of Programming Languages and Tools PLATEAU '14, 2014, pp. 55-56.

[34] S. Vosinakis, P. Koutsabasis, and G. Anastassakis, "A Platform for Teaching Logic Programming Using Virtual Worlds," in 2014 IEEE 14th International Conference on Advanced Learning Technologies, 2014, pp. 657-661.

[35] R. Swamidurai, "Improving undergraduate students programming skills through Collaborative Adversarial Pair Learning," in IEEE SOUTHEASTCON 2014, 2014, pp. 14.
[36] R. Duque, D. Gómez-Pérez, A. Nieto-Reyes, and C. Bravo, "Analyzing collaboration and interaction in learning environments to form learner groups," Comput. Human Behav., vol. 47, pp. 42-49, Jun. 2015.

[37] P. Awasthi and I. H. Hsaio, "INSIGHT: A semantic visual analytics for programming discussion forums," in CEUR Workshop Proceedings, 2015, vol. 1518, pp. 24-31.

[38] A. L. Santos, "Collaborative course project for practicing component-based software engineering," in Proceedings of the 15th Koli Calling Conference on Computing Education Research - Koli Calling '15, 2015, pp. 142146.

[39] A. Nylén, N. Thota, A. Eckerdal, P. Kinnunen, M. Butler, and M. Morgan, "Multidimensional analysis of creative coding MOOC forums," in Proceedings of the 15th Koli Calling Conference on Computing Education Research - Koli Calling '15, 2015, pp. 137-141.

[40] A. Herala, E. Vanhala, A. Knutas, and J. Ikonen, "Teaching programming with flipped classroom method," in Proceedings of the 15th Koli Calling Conference on Computing Education Research - Koli Calling '15, 2015, pp. 165-166.

[41] C. Latulipe, N. B. Long, and C. E. Seminario, "Structuring Flipped Classes with Lightweight Teams and Gamification," in Proceedings of the 46th ACM Technical Symposium on Computer Science Education SIGCSE' '15, 2015, pp. 392-397.

[42] T. Ndabvonga-Dongo and A. H. Reed, "Enhancing software development in the MIS curriculum using pair programming," in 2015 Americas Conference on Information Systems, AMCIS 2015, 2015, pp. 1-9.

[43] S. Azmi, N. A. Iahad, and N. Ahmad, "Gamification in online collaborative learning for programming courses: A literature review," ARPN J. Eng. Appl. Sci., vol. 10, no. 23, pp. 18087-18094, 2015.

[44] Y. Hayashi, K.-I. Fukamachi, and H. Komatsugawa, "Collaborative Learning in Computer Programming Courses That Adopted the Flipped Classroom," in 2015 International Conference on Learning and Teaching in Computing and Engineering, 2015, pp. 209-212.

[45] P. J. Guo, J. White, and R. Zanelatto, "Codechella: Multi-user program visualizations for real-time tutoring and collaborative learning," in 2015 IEEE Symposium on Visual Languages and Human-Centric Computing (VL/HCC), 2015, pp. 79-87.

[46] E. Stankov, M. Jovanov, B. Kostadinov, and A. Madevska Bogdanova, "A new model for 
El trabajo colaborativo como estrategia didáctica para la enseñanza/aprendizaje de la programación: una revisión sistemática de literatura

collaborative learning of programming using source code similarity detection," in 2015 IEEE Global Engineering Education Conference (EDUCON), 2015, pp. 709-715.

[47] K. Sullivan, J. R. Byrne, N. Bresnihan, K. O'Sullivan, and B. Tangney, "CodePlusDesigning an after school computing programme for girls," in 2015 IEEE Frontiers in Education Conference (FIE), 2015, pp. 1-5.

[48] L.-K. Lee, O. Au, R. So, and N.-I. Wu, "Being Well-Prepared for Regular PairProgramming Helps At-Risk Students," in 2016 International Symposium on Educational Technology (ISET), 2016, pp. 65-68.

[49] B. Zhong, Q. Wang, and J. Chen, "The impact of social factors on pair programming in a primary school," Comput. Human Behav., vol. 64, pp. 423-431, 2016.

[50] A. Eck, L.-K. Soh, and D. F. Shell, "Investigating Differences in Wiki-based Collaborative Activities between Student Engagement Profiles in CS1," in Proceedings of the 47th ACM Technical Symposium on Computing Science Education - SIGCSE '16, 2016, pp. 36-41.

[51] C. Kussmaul, H. H. Hu, and C. Mayfield, "Guiding Students to Discover CS Concepts and Develop Process Skills using POGIL (Abstract Only)," in Proceedings of the 47th ACM Technical Symposium on Computing Science Education - SIGCSE '16, 2016, pp. $712-712$.

[52] K. Thongkoo, "Mobile Learning Development for Supporting Computer Programming Skills," in Lecture Notes in Electrical Engineering, vol. 391, 2016, pp. 181-186.

[53] X. Wu and X. Feng, "A case study of projectbased industrial collaborative learning courses for teaching high school programming development in China," in 10th International Multi-Conference on Society, Cybernetics and Informatics, Proceedings, 2016, pp. 148-151.

[54] M. Coccoli, P. Maresca, and L. Stanganelli, "Teaching Computer Programming Through Hands-on Labs on Cognitive Computing," in Proceedings - DMS 2016: 22nd International Conference on Distributed Multimedia Systems, 2016, pp. 158-164.

[55] G.-J. Hwang, Z.-Y. Liang, and H.-Y. Wang, "An Online Peer Assessment-Based Programming Approach to Improving
Students' Programming Knowledge and Skills," in 2016 International Conference on Educational Innovation through Technology (EITT), 2016, pp. 81-85.

[56] M. Nawahdah and D. Taji, "Investigating students' behavior and code quality when applying pair-programming as a teaching technique in a Middle Eastern society," in 2016 IEEE Global Engineering Education Conference (EDUCON), 2016, vol. 10-13Apri, pp. 32-39.

[57] J. P. Ucan, O. S. Gomez, and R. A. Aguilar, "Assessment of software defect detection efficiency and cost through an intelligent collaborative virtual environment," IEEE Lat. Am. Trans., vol. 14, no. 7, pp. 33643369, Jul. 2016.

[58] C. Patek and A. Chattopadhyay, "Can Undergraduate Computing Research Be Student-Driven? (Abstract Only)," in Proceedings of the 2017 ACM SIGCSE Technical Symposium on Computer Science Education - SIGCSE'17, 2017, pp. 715-715.

[59] M. D. Sakhumuzi and O. K. Emmanuel, "Student perception of the contribution of Hackathon and collaborative learning approach on computer programming pass rate," in 2017 Conference on Information Communication Technology and Society (ICTAS), 2017, pp. 1-5.

[60] W. C. Hsu and H. C. K. Lin, "Impact of Applying WebGL Technology to Develop a Web Digital Game-Based Learning System for Computer Programming Course in Flipped Classroom," in Proceedings - 5th International Conference on Educational Innovation through Technology, EITT 2016, 2017, pp. 64-69.

[61] S. Ghorashi and C. Jensen, "Integrating Collaborative and Live Coding for Distance Education," Computer (Long. Beach. Calif)., vol. 50, no. 5, pp. 27-35, May 2017.

[62] A. Serenko, N. Bontis, L. Booker, K. Sadeddin, and T. Hardie, "A scientometric analysis of knowledge management and intellectual capital academic literature (1994-2008)," J. Knowl. Manag., vol. 14, no. 1, pp. 3-23, Feb. 2010.

[63] R. Wieringa, N. Maiden, N. Mead, and C. Rolland, "Requirements engineering paper classification and evaluation criteria: a proposal and a discussion," Requir. Eng., vol. 11, no. 1, pp. 102-107, Mar. 2006. 